\title{
Modelling operational expenditure savings for the implementation of autonomous networks
}

\author{
Sander Spek, Vânia Gonçalves, Olivier Rits \\ iMinds-SMIT, Vrije Universiteit Brussel \\ Pleinlaan 2, 1050 Brussels, Belgium \\ \{alexander.spek, vania.goncalves\}@vub.ac.be, \\ olivier.rits@iminds.be
}

\author{
Zwi Altman, Christian Destré \\ Orange Labs \\ Issy Les Moulineaux, France \\ \{zwi.altman, christian.destre\}@orange.com
}

\begin{abstract}
Developments in autonomic network management offer many promises, but its economic benefits are hard to assess. This paper proposes a method to calculate the OPEX gains of a typical network on the basis of a scenario, for which a management framework and autonomous mechanisms have been developed. It makes use of a novel approach, a Toy model, taking into account expert opinions as well as simulation results. The new approach allows assessment of the OPEX impact of both individual mechanisms as well as the overall impact, which, in the present scenario results in an expected OPEX saving of 11 to 13 per cent.
\end{abstract}

Keywords-autonomic networking; self-x; opex; business modelling; implementation;

\section{INTRODUCTION}

An important driver for autonomic network management is the need to simplify network operation and alleviate the burden of network management in a highly heterogeneous network ecosystem. Autonomicity is achieved by introducing self-x features or control loops in the service and network management systems that have to be redesigned to support such a new paradigm. A Unified Management Framework (UMF) has been specified in the form of functional blocks, interfaces and implementation guidelines to ensure the trustworthy integration (plug and play), operation and interworking (conflict avoidance and knowledge sharing) of multiple autonomic control loops for managing networks and services. ${ }^{1}$ A set of autonomic solutions, i.e. self-x functions, methods and algorithms with the aim to solve specific telecom operator problems, were designed according to the functional specifications and implementation guidelines. These are denoted as Network Empowerment Mechanisms (NEMs). Three UMF core blocks have been specified: (1) Governance, which aims to provide a human operator a policy-based mechanism for controlling the network/NEMs from a high level business point of view; (2) Coordination, which is responsible for the proper inter-operation of the NEMs: conflict avoidance between competing NEMs, stability enforcement of inter-working NEMs and the joint optimization of tightly coupled NEMs; (3) Knowledge, which is responsible for the unified management of information and knowledge in the UMF system: optimized collection, aggregation, storage, processing and distribution of information. The first large-scale deployment of self-x

1 As part of the UniverSelf FP7 European research project [1]. features are related to the Self-Organizing Network (SON) in 3GPP Long Term Evolution (LTE) networks. SON is considered as a means to decrease networks operation and management costs by increasing the level of automation. Existing implementations of 3GPP SON features show some limitations, e.g. for multi-vendor SON interworking, and coordination of multiple SON features. SON within UMF will allow taking full advantage from the SON technology.

The capability to estimate the potential OPEX gains brought about by self-x functionalities is essential for prioritising development and implementation of the most useful ones, and for establishing a global deployment and operation strategy of the network. Qualitative business impacts have been assessed before, e.g. in [2], but quantitative OPEX statements have not been made. This paper proposes a Toy model to do so. In the next section, a scenario is described, which serves as an example for demonstrating the method. This method itself is presented in section III and the results of its application to the scenario are described in section IV. Finally, in section $\mathrm{V}$ a discussion and concluding remarks on the method and the results are provided.

\section{SCENARIO}

This paper focuses on OPEX modeling for a scenario developed entitled: "SON and SON collaboration according to operator policies" [3]. The scenario focuses on three research challenges identified as essential for benefitting from the SON paradigm: (1) design novel SON functionalities for future radio access networks, (2) design solutions for coordinated operation of SON functionalities, and (3) integrate SON operation in UMF. Within the scenario, SON functionalities have been designed to operate in LTE, LTE-Advanced and WLAN networks, following the NEMs' specifications. Examples are load balancing, coverage and capacity optimization, and cell outage compensation. Solution for conflict resolution has been designed as a pre-deployment phase of the SONs. When instability occurs at run time, both control and optimization based solutions have been developed as part of the Joint Optimization used by the UMF coordination block (e.g. [3][4][5][6]). Three UMF compliant prototypes have been designed following the UMF framework: (1) SON coordination in a LTE-Advanced network with relays, (2) SON coordinated optimization of inter-cell interference coordination and coverage and capacity optimization in a LTE network, and (3) cell outage compensation in a WLAN.

978-1-4799-3083-8/14/\$31.00 @ 2014 IEEE 


\section{METHOD}

\section{A. Overview}

The goal of the Toy model method is threefold: 1) to provide a pragmatic, simple and explicit tool to compute the attainable OPEX reductions based on NEM numerical performance evaluations; 2) to reason about the aggregate findings for scenarios; and, 3) to deduce trends and assertions about the potential of the technologies to impact OPEX. It is not obvious to identify on the basis of the scenario and the UMF perspectives how OPEX would be impacted. There are several dimensions to consider. Firstly, the NEMs and methods are designed and developed for solving operational problems by providing self-x features. The direct impact related to these operations is easily identified. However, impact on other facets of OPEX has to be identified as well as the link between performance of these features and OPEX. Secondly, the impact of the adoption and use of UMF is larger than the operations that are directly impacted by self-x features. In that respect, the integration and management tool life cycles are also concerned. Finally, we stress that OPEX is a complex notion with many aspects. When referring to OPEX many cost categories need to be identified.

To capture all these dimensions, we propose to identify the OPEX impact based on the processes of the TMF Business Process Framework (eTOM) [7]. An exhaustive analysis of the processes and their definition helps to identify where NEM, or UMF adoption have impact. The proposed method is depicted in Fig. 1. It uses NEM metric results provided by NEM developers as a starting point, which are mapped onto eTOM process flows (branch A). An expert mapping that was previously performed in the project in the form of QFD (see e.g. [8]) constitutes an a priori analysis (branch B, see [9]). Finally, in Branch $\mathrm{C}$, the two earlier inputs converge in a mapping on OPEX categories, which on its turn can provide the OPEX impact per NEM.

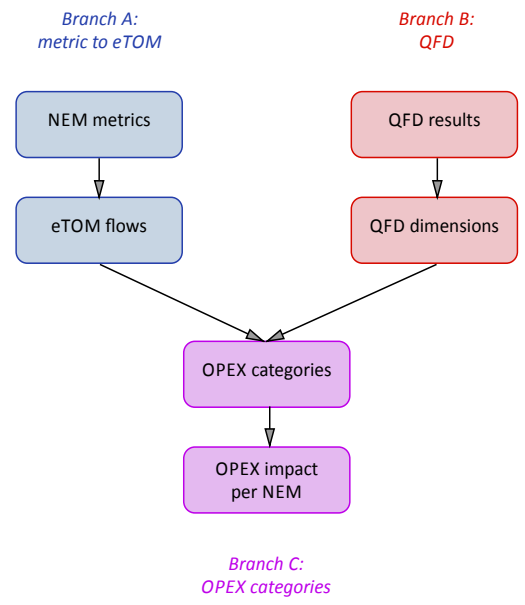

Fig. 1. A high-level overview of the Toy model

The metric results that form the starting point for our analysis are presented in Subsection B. The QFD scores that had been provided in the a priori analysis are used here. For the other three types of expert inputs (metric to eTOM process mapping, eTOM process to category mapping, QFD to category mapping), these results are presented in Subsection C. For the relative sizes of OPEX categories, a reference from literature has been used, which is explained in detail in Subsection $\mathrm{C}$ as well.

\section{B. Inputs}

The NEM owners have identified the metrics and provided simulation results, which serve as in input in this exercise. An overview of the NEMs together with their metrics is presented in TABLE I. It shows the metrics' best cases, bases cases and gains.

The results obtained by the deployment of the NEM 3 Inter-Cell Interference Coordination (i.e. best case for NEM 3) are compared with the results obtained by the deployment of NEM 4 - Coverage and Capacity Optimization (i.e. base case for NEM 3). In the same way, referring to NEM 4, the results obtained by the deployment of NEM 4 (i.e. best case for NEM 4) are compared with the results obtained by the deployment of NEM 3 (i.e. base case for NEM 4). NEM 3 presents gains on inter-cell Interference and SINR while there are losses on cell throughput and user throughput. The opposite remarks are valid while considering NEM 4 instead.

NEM 28 - Prediction-based Load Balancing metrics refer to outage derived from the number of dropped mobile devices and user satisfaction as the delta between the actual and predicted user satisfaction. An algorithmic framework is used for the proactive load balancing of user decision-making requests, assuming reconfigurable mobile devices (base case) and autonomous mobile devices (best case). The obtained results show the gains of the presented framework in terms of the number of dropped user requests for autonomous mobile devices.

For NEM 32 - Self-healing mechanism for Cell Outage Management, a fuzzy inference system is considered and the values presented refer to worst performance results (base case) and the best performance results. These indicative results have been simulated for a $3 \times 3$ grid ( 9 access points (APs)) and for a varying number of mobile terminals. The algorithm uses a fuzzy inference system with the following inputs: a) the compensating AP's load and b) a metric of the overlapping area that each compensating AP creates with the neighbouring APs.

NEM 34 - LTE Load Balancing and NEM 35 - LTE Tilt Optimization adapts the cell and/or antenna parameter in such a way that cell resources and coverage area are best adapted to serve the user traffic. The achieved performance gains do strongly depend on the amount and distribution of traffic. Very large performance gains can in particular be achieved with diverse traffic loads, varying between neighbouring cells and at an overall traffic load level which is in the medium-high-load to high-load range. In contrast the NEM gains are very low for very uniform traffic distributions and at traffic load levels of either a basically empty system or of a complete overload everywhere. For realistic traffic modelling and diversity, the typical NEM gains are in the order of around $10 \%$. The results below are of macro-cellular studies without traffic hotspots.

As for NEM $46-G P$ Coverage, a static coverage is used as a base case for comparison, where femtocells are configured 
with a fixed pilot power of $-29 \mathrm{dBm}$. The results provided represent the performance under overloaded conditions. The load supported by the femtocells with the coverage algorithm is approximately $20 \%$ higher than the load supported by the fixed coverage deployment. The number of macrocell user mobility requests with the coverage algorithm is lower compared with the fixed coverage deployment. The number of mobility events between macrocells and femtocell is slightly higher due to the femtocells creating temporary coverage holes when changing coverage when load balancing, but this is not significant.

TABLE I. METRIC SIMULATION RESULTS PER NEM

\begin{tabular}{|c|c|c|c|c|}
\hline NEM & Metric & $\begin{array}{l}\text { Best } \\
\text { Case }\end{array}$ & $\begin{array}{l}\text { Base } \\
\text { Case }\end{array}$ & Gains \\
\hline \multirow[t]{4}{*}{3} & Inter-cell interference $(\mathrm{dBm})$ & $-47,3$ & $-46,2$ & $2 \%$ \\
\hline & SINR $(\mathrm{dB})$ & 12,8052 & 12,3399 & $4 \%$ \\
\hline & Cell throughput (Kbps) & 7720 & 12440 & $-38 \%$ \\
\hline & User throughput (Kbps) & 643 & 1040 & $-38 \%$ \\
\hline \multirow[t]{3}{*}{4} & SINR $(\mathrm{dB})$ & 12,3399 & 12,8052 & $-4 \%$ \\
\hline & Cell throughput (Kbps) & 12440 & 7720 & $61 \%$ \\
\hline & User throughput (Kbps) & 1040 & 643 & $62 \%$ \\
\hline \multirow[t]{2}{*}{28} & Outage & $9,3 \%$ & $11,1 \%$ & $16 \%$ \\
\hline & User satisfaction & 0 & 0,06 & $100 \%$ \\
\hline \multirow[t]{3}{*}{32} & Interference indicator & 0,06 & 1,72 & $97 \%$ \\
\hline & Load fairness index & 0,581 & 0,709 & $18 \%$ \\
\hline & Ratio of recovered terminals & $6 / 6$ & $1 / 1$ & $0 \%$ \\
\hline 34 & Cell throughput (Mbps/cell) & 10,6 & 10,3 & $3 \%$ \\
\hline \multirow[t]{2}{*}{35} & Cell throughput (Mbps/cell) & 20,9 & 19 & $10 \%$ \\
\hline & Cell edge throughput (Mbps/cell) & 1,6 & 1,2 & $33 \%$ \\
\hline \multirow[t]{3}{*}{46} & $\begin{array}{l}\text { Average load supported by femtocells } \\
\text { (Erlangs) }\end{array}$ & 80,91 & 64,89 & $25 \%$ \\
\hline & $\begin{array}{l}\text { Average macrocell user mobility } \\
\text { requests per pass }\end{array}$ & 0,1835 & 3,9998 & $95 \%$ \\
\hline & $\begin{array}{l}\text { Average femto } \leftrightarrow \text { macro mobility } \\
\text { events to experienced by a femtocell } \\
\text { user per hour }\end{array}$ & 0,0938 & 0,0627 & $-50 \%$ \\
\hline
\end{tabular}

\section{Conversion Tables}

This subsection contains the mapping tables used in the Toy model generated by expert inputs (metric to eTOM process mapping, eTOM process to category mapping, QFD to category mapping) as well as the cost category mapping that has been taken from literature. Starting with the latter, it specifies the different OPEX components of a typical network operator, as well as the relative sizes. Several overviews of cost categories for OPEX for telecom operators exist, for example by Verbrugge et al. [10], which is extended by Cid et al. [11]. While the cost categories proposed are very suitable for modelling, there is no information of the relative sizes of these categories for a typical network operator, be it fixed or mobile. Several sources provide alternative OPEX categories while also providing information on the relative sizes of the categories, e.g. [12][13][14][15][16]. In this paper, the categories of a 2011 white paper of Deloitte [17] will be used, since it is sufficiently fine-grained, it focuses on wireless carriers only, and it is the most recent of the studies. Where comparisons are possible, the results of this study seem coherent with the results of the others. Moreover, for most categories, this study provides ranges rather than possibly inaccurate fixed values. Cost categories, with the corresponding relative sizes, are presented in columns
'Minimum' and 'Maximum' of TABLE II. When summing up the minimum values from the range, one arrives at a total OPEX of $100 \%$. This is not workable, since it means that for one category to be larger than the minimum, one other needs to drop below the minimum. Therefore, we have taken the middle of the range $(108 \%)$ and normalized over that. That is presented in TABLE II. under columns 'Corrected minimum' and 'Corrected maximum.

TABLE II. RELATIVE SIZES OF OPEX CATEGORIES

\begin{tabular}{|c|c|c|c|c|c|}
\hline $\begin{array}{l}\text { OPEX } \\
\text { group }\end{array}$ & OPEX category & 哥 & $\sum_{\Sigma}^{\Xi}$ & 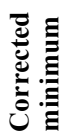 & 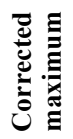 \\
\hline $\begin{array}{l}\text { Non-process } \\
\text { costs }\end{array}$ & $\begin{array}{l}\text { e.g. interconnection fees, taxes, } \\
\text { CPE, uncollectible items. }\end{array}$ & $35 \%$ & $35 \%$ & $32 \%$ & $32 \%$ \\
\hline \multirow{4}{*}{$\begin{array}{l}\text { Support- } \\
\text { process costs }\end{array}$} & Marketing & $10 \%$ & $12 \%$ & $9 \%$ & $11 \%$ \\
\hline & IT (excluding billing) & $4 \%$ & $6 \%$ & $4 \%$ & $6 \%$ \\
\hline & Finance and management & $3 \%$ & $5 \%$ & $3 \%$ & $5 \%$ \\
\hline & HR, benefits and others & $3 \%$ & $5 \%$ & $3 \%$ & $5 \%$ \\
\hline \multirow{4}{*}{$\begin{array}{l}\text { Operational- } \\
\text { process costs }\end{array}$} & Sales & $18 \%$ & $20 \%$ & $17 \%$ & $19 \%$ \\
\hline & Customer service & $10 \%$ & $12 \%$ & $9 \%$ & $11 \%$ \\
\hline & Billing (Order to cash) & $2 \%$ & $4 \%$ & $2 \%$ & $4 \%$ \\
\hline & Network and maintenance costs & $15 \%$ & $17 \%$ & $14 \%$ & $16 \%$ \\
\hline \multicolumn{2}{|l|}{ Sum } & $100 \%$ & $116 \%$ & $93 \%$ & $107 \%$ \\
\hline
\end{tabular}

As explained previously, the data in the other conversion tables are based on an expert survey. In total eight experts have provided their values, and the averaged results have been structured into three mapping tables.

The 'Metrics to eTOM flows' tables map the impact of the different NEM metrics onto the eTOM flows of the two most relevant processes: Customer Centric E2E Business Process and Network Centric E2E Business Process. The results are presented in TABLE III. and TABLE IV. respectively. The mapping of the eTOM process flows to the OPEX categories is presented in TABLE V. , while the mapping of QFD dimensions is displayed in TABLE VI.

TABLE III. METRIC TO ETOM FLOW CUSTOMER CENTRIC E2E BuSINESS PROCESS

\begin{tabular}{|c|c|c|c|c|c|c|c|c|}
\hline \multicolumn{2}{|c|}{$\begin{array}{l}\text { Metric to eTOM flow } \\
\text { Customer Centric E2E } \\
\text { Business Process }\end{array}$} & \multirow{2}{*}{ 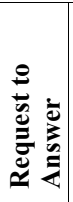 } & \multirow{2}{*}{ 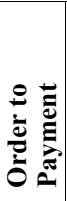 } & \multirow{2}{*}{ 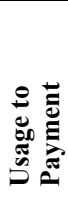 } & \multirow{2}{*}{ 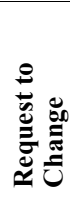 } & \multirow{2}{*}{ 。 } & \multirow{2}{*}{ 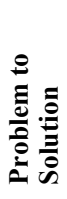 } & \multirow{2}{*}{ 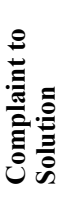 } \\
\hline NEM & Metric & & & & & & & \\
\hline \multirow[t]{4}{*}{3} & Inter-cell interference & $0 \%$ & $0 \%$ & $0 \%$ & $1 \%$ & $0 \%$ & $5 \%$ & $2 \%$ \\
\hline & SINR & $0 \%$ & $0 \%$ & $0 \%$ & $1 \%$ & $0 \%$ & $6 \%$ & $2 \%$ \\
\hline & Cell throughput & $0 \%$ & $0 \%$ & $0 \%$ & $1 \%$ & $0 \%$ & $5 \%$ & $1 \%$ \\
\hline & User throughput & $0 \%$ & $0 \%$ & $0 \%$ & $1 \%$ & $0 \%$ & $5 \%$ & $1 \%$ \\
\hline \multirow[t]{3}{*}{4} & SINR & $0 \%$ & $0 \%$ & $1 \%$ & $2 \%$ & $0 \%$ & $6 \%$ & $2 \%$ \\
\hline & Cell throughput & $0 \%$ & $0 \%$ & $1 \%$ & $2 \%$ & $0 \%$ & $6 \%$ & $2 \%$ \\
\hline & User throughput & $0 \%$ & $0 \%$ & $1 \%$ & $2 \%$ & $0 \%$ & $7 \%$ & $3 \%$ \\
\hline \multirow[t]{2}{*}{28} & Outage & $0 \%$ & $0 \%$ & $0 \%$ & $1 \%$ & $0 \%$ & $12 \%$ & $2 \%$ \\
\hline & User satisfaction & $0 \%$ & $0 \%$ & $0 \%$ & $1 \%$ & $0 \%$ & $6 \%$ & $1 \%$ \\
\hline \multirow[t]{3}{*}{32} & Interference & $0 \%$ & $0 \%$ & $0 \%$ & $1 \%$ & $0 \%$ & $8 \%$ & $3 \%$ \\
\hline & Load fairness index & $0 \%$ & $0 \%$ & $0 \%$ & $1 \%$ & $0 \%$ & $11 \%$ & $1 \%$ \\
\hline & Reconnected MTs & $0 \%$ & $0 \%$ & $0 \%$ & $1 \%$ & $0 \%$ & $19 \%$ & $9 \%$ \\
\hline 34 & Cell throughput & $0 \%$ & $0 \%$ & $0 \%$ & $1 \%$ & $0 \%$ & $4 \%$ & $1 \%$ \\
\hline \multirow[t]{2}{*}{35} & Cell throughput & $0 \%$ & $0 \%$ & $0 \%$ & $1 \%$ & $0 \%$ & $3 \%$ & $0 \%$ \\
\hline & Cell edge throughput & $0 \%$ & $0 \%$ & $0 \%$ & $1 \%$ & $0 \%$ & $4 \%$ & $0 \%$ \\
\hline
\end{tabular}




\begin{tabular}{|c|c|c|c|c|c|c|c|c|}
\hline \multirow[t]{3}{*}{46} & $\begin{array}{l}\text { Average load } \\
\text { supported by } \\
\text { femtocells }\end{array}$ & $0 \%$ & $0 \%$ & $0 \%$ & $1 \%$ & $0 \%$ & $7 \%$ & $6 \%$ \\
\hline & $\begin{array}{l}\text { Average macrocell } \\
\text { user mobility requests } \\
\text { per pass }\end{array}$ & $0 \%$ & $0 \%$ & $0 \%$ & $1 \%$ & $0 \%$ & $2 \%$ & $0 \%$ \\
\hline & $\begin{array}{l}\text { Average femto } \leftrightarrow \\
\text { macro mobility events } \\
\text { to experienced by a } \\
\text { femtocell user per } \\
\text { hour }\end{array}$ & $0 \%$ & $0 \%$ & $0 \%$ & $1 \%$ & $0 \%$ & $4 \%$ & $2 \%$ \\
\hline
\end{tabular}

TABLE IV. METRIC TO ETOM FLOW NETWORK CENTRIC E2E BUSINESS PROCESS

\begin{tabular}{|c|c|c|c|c|c|c|c|}
\hline \multirow{2}{*}{\multicolumn{2}{|c|}{$\begin{array}{l}\text { Metric to eTOM flow } \\
\text { Network Centric E2E Business } \\
\text { Process }\end{array}$}} & \multirow{3}{*}{$\begin{array}{l}0 \\
0\end{array}$} & \multirow{3}{*}{ 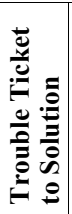 } & \multirow{3}{*}{ 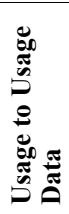 } & \multirow{3}{*}{ 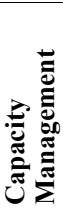 } & \multirow{3}{*}{ 窇 } & \multirow{3}{*}{ 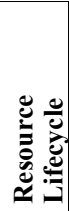 } \\
\hline & & & & & & & \\
\hline NEM & Metric & & & & & & \\
\hline \multirow[t]{4}{*}{3} & Inter-cell interference & $2 \%$ & $6 \%$ & $0 \%$ & $14 \%$ & $2 \%$ & $8 \%$ \\
\hline & SINR & $2 \%$ & $6 \%$ & $0 \%$ & $13 \%$ & $3 \%$ & $8 \%$ \\
\hline & Cell throughput & $2 \%$ & $7 \%$ & $0 \%$ & $14 \%$ & $2 \%$ & $8 \%$ \\
\hline & User throughput & $2 \%$ & $7 \%$ & $0 \%$ & $14 \%$ & $2 \%$ & $8 \%$ \\
\hline \multirow[t]{3}{*}{4} & SINR & $1 \%$ & $6 \%$ & $0 \%$ & $17 \%$ & $3 \%$ & $10 \%$ \\
\hline & Cell throughput & $2 \%$ & $6 \%$ & $0 \%$ & $18 \%$ & $3 \%$ & $10 \%$ \\
\hline & User throughput & $2 \%$ & $7 \%$ & $0 \%$ & $18 \%$ & $3 \%$ & $10 \%$ \\
\hline \multirow[t]{2}{*}{28} & Outage & $1 \%$ & $9 \%$ & $0 \%$ & $8 \%$ & $3 \%$ & $11 \%$ \\
\hline & User satisfaction & $1 \%$ & $4 \%$ & $0 \%$ & $5 \%$ & $2 \%$ & $9 \%$ \\
\hline \multirow[t]{3}{*}{32} & Interference & $1 \%$ & $3 \%$ & $0 \%$ & $4 \%$ & $2 \%$ & $9 \%$ \\
\hline & Load fairness index & $1 \%$ & $7 \%$ & $0 \%$ & $3 \%$ & $3 \%$ & $13 \%$ \\
\hline & $\begin{array}{l}\text { Percentage of reconnected } \\
\text { MTs }\end{array}$ & $1 \%$ & $14 \%$ & $0 \%$ & $8 \%$ & $3 \%$ & $13 \%$ \\
\hline 34 & Cell throughput & $1 \%$ & $4 \%$ & $0 \%$ & $12 \%$ & $2 \%$ & $10 \%$ \\
\hline \multirow[t]{2}{*}{35} & Cell throughput & $1 \%$ & $4 \%$ & $0 \%$ & $19 \%$ & $2 \%$ & $9 \%$ \\
\hline & Cell edge throughput & $1 \%$ & $4 \%$ & $0 \%$ & $19 \%$ & $2 \%$ & $9 \%$ \\
\hline \multirow[t]{3}{*}{46} & $\begin{array}{l}\text { Average load supported by } \\
\text { femtocells }\end{array}$ & $2 \%$ & $2 \%$ & $0 \%$ & $14 \%$ & $2 \%$ & $10 \%$ \\
\hline & $\begin{array}{l}\text { Average macrocell user } \\
\text { mobility requests per pass }\end{array}$ & $2 \%$ & $1 \%$ & $0 \%$ & $6 \%$ & $2 \%$ & $6 \%$ \\
\hline & $\begin{array}{l}\text { Average femto } \leftrightarrow \text { macro } \\
\text { mobility events to } \\
\text { experienced by a femtocell } \\
\text { user per hour } \\
\end{array}$ & $2 \%$ & $3 \%$ & $0 \%$ & $6 \%$ & $2 \%$ & $6 \%$ \\
\hline
\end{tabular}

TABLE V. MAPPING OF ETOM PROCESS FLOWS TO OPEX CATEGORIES

\begin{tabular}{|c|c|c|c|c|c|c|c|c|c|}
\hline & 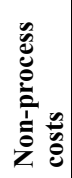 & 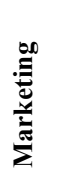 & 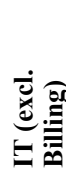 & 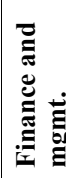 & 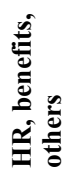 & $\frac{\tilde{\sigma}}{\tilde{E}}$ & 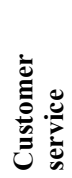 & 里 & 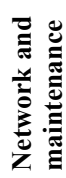 \\
\hline $\begin{array}{l}\text { Request to } \\
\text { Answer }\end{array}$ & $0 \%$ & $3 \%$ & $1 \%$ & $1 \%$ & $5 \%$ & $14 \%$ & $17 \%$ & $0 \%$ & $1 \%$ \\
\hline $\begin{array}{l}\text { Order to } \\
\text { Payment }\end{array}$ & $0 \%$ & $0 \%$ & $5 \%$ & $1 \%$ & $1 \%$ & $0 \%$ & $5 \%$ & $11 \%$ & $1 \%$ \\
\hline $\begin{array}{l}\text { Usage to } \\
\text { Payment }\end{array}$ & $0 \%$ & $0 \%$ & $5 \%$ & $2 \%$ & $2 \%$ & $0 \%$ & $5 \%$ & $21 \%$ & $1 \%$ \\
\hline $\begin{array}{l}\text { Request to } \\
\text { Change }\end{array}$ & $0 \%$ & $0 \%$ & $5 \%$ & $1 \%$ & $2 \%$ & $1 \%$ & $6 \%$ & $0 \%$ & $3 \%$ \\
\hline $\begin{array}{l}\text { Termination to } \\
\text { Confirmation }\end{array}$ & $0 \%$ & $0 \%$ & $3 \%$ & $2 \%$ & $2 \%$ & $0 \%$ & $7 \%$ & $2 \%$ & $2 \%$ \\
\hline $\begin{array}{l}\text { Problem to } \\
\text { Solution }\end{array}$ & $0 \%$ & $2 \%$ & $3 \%$ & $1 \%$ & $8 \%$ & $2 \%$ & $17 \%$ & $0 \%$ & $9 \%$ \\
\hline
\end{tabular}

\begin{tabular}{|l|c|c|c|c|c|c|c|c|c|}
\hline $\begin{array}{l}\text { Complaint to } \\
\text { Solution }\end{array}$ & $0 \%$ & $2 \%$ & $3 \%$ & $1 \%$ & $9 \%$ & $2 \%$ & $17 \%$ & $1 \%$ & $2 \%$ \\
\hline $\begin{array}{l}\text { Production } \\
\text { Order to } \\
\text { Acceptance }\end{array}$ & $0 \%$ & $1 \%$ & $4 \%$ & $1 \%$ & $4 \%$ & $2 \%$ & $0 \%$ & $0 \%$ & $8 \%$ \\
\hline $\begin{array}{l}\text { Trouble Ticket to } \\
\text { Solution }\end{array}$ & $0 \%$ & $0 \%$ & $3 \%$ & $0 \%$ & $5 \%$ & $2 \%$ & $5 \%$ & $2 \%$ & $14 \%$ \\
\hline $\begin{array}{l}\text { Usage to Usage } \\
\text { Data }\end{array}$ & $1 \%$ & $0 \%$ & $2 \%$ & $0 \%$ & $2 \%$ & $0 \%$ & $2 \%$ & $10 \%$ & $6 \%$ \\
\hline $\begin{array}{l}\text { Capacity } \\
\text { Management }\end{array}$ & $1 \%$ & $0 \%$ & $0 \%$ & $1 \%$ & $3 \%$ & $0 \%$ & $10 \%$ & $0 \%$ & $25 \%$ \\
\hline $\begin{array}{l}\text { Service Lifecycle } \\
\text { Management }\end{array}$ & $0 \%$ & $3 \%$ & $2 \%$ & $1 \%$ & $3 \%$ & $3 \%$ & $2 \%$ & $2 \%$ & $10 \%$ \\
\hline $\begin{array}{l}\text { Resource } \\
\text { Lifecycle } \\
\text { Management }\end{array}$ & $0 \%$ & $3 \%$ & $1 \%$ & $2 \%$ & $5 \%$ & $3 \%$ & $2 \%$ & $2 \%$ & $19 \%$ \\
\hline
\end{tabular}

TABLE VI. MAPPING OF QFD DIMENSIONS TO OPEX CATEGORIES

\begin{tabular}{|c|c|c|c|c|c|c|c|c|c|c|}
\hline $\begin{array}{l}\text { QFD } \\
\text { dimensions }\end{array}$ & 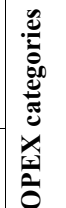 & 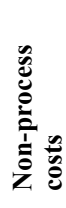 & 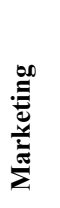 & 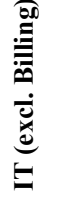 & 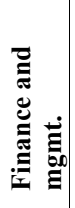 & 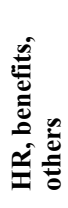 & $\frac{\mathscr{e}}{\pi}$ & 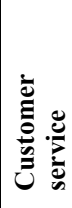 & 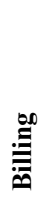 & 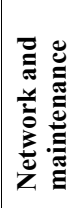 \\
\hline \multicolumn{2}{|c|}{$\begin{array}{l}\text { New services and } \\
\text { revenues }\end{array}$} & $0 \%$ & $11 \%$ & $1 \%$ & $0 \%$ & $5 \%$ & $11 \%$ & $5 \%$ & $1 \%$ & $5 \%$ \\
\hline \multicolumn{2}{|c|}{ Cost of adoption } & $0 \%$ & $0 \%$ & $6 \%$ & $0 \%$ & $3 \%$ & $4 \%$ & $4 \%$ & $1 \%$ & $13 \%$ \\
\hline \multicolumn{2}{|l|}{ Energy } & $3 \%$ & $0 \%$ & $1 \%$ & $0 \%$ & $4 \%$ & $1 \%$ & $2 \%$ & $0 \%$ & $9 \%$ \\
\hline \multicolumn{2}{|c|}{$\begin{array}{l}\text { Operation and } \\
\text { maintenance } \\
\text { personnel }\end{array}$} & $1 \%$ & $0 \%$ & $5 \%$ & $0 \%$ & $12 \%$ & $3 \%$ & $8 \%$ & $0 \%$ & $33 \%$ \\
\hline \multicolumn{2}{|l|}{$\begin{array}{l}\text { General } \\
\text { autonomics } \\
\text { impact }\end{array}$} & $0 \%$ & $0 \%$ & $5 \%$ & $0 \%$ & $9 \%$ & $2 \%$ & $13 \%$ & $0 \%$ & $28 \%$ \\
\hline \multicolumn{2}{|c|}{$\begin{array}{l}\text { Visits / } \\
\text { transportation }\end{array}$} & $1 \%$ & $4 \%$ & $0 \%$ & $1 \%$ & $8 \%$ & $8 \%$ & $6 \%$ & $0 \%$ & $26 \%$ \\
\hline
\end{tabular}

\section{Model Results}

This section presents the results of the Toy method applied to the described scenario. In Subsection A, the OPEX impacts for the individual NEMs are presented, while in Subsection B the individual results are aggregated into a general OPEX impact for the scenario.

\section{A. Individual NEM Results}

When calculating the OPEX savings according to the proposed method and using the tables provided in the previous subsections, one arrives at the results presented in TABLE VII. Some NEMs have a low impact of less than one per cent (which can still be significant for an operator), while others go as far as almost five per cent. Normally, an operator will not implement the UMF framework just for executing one NEM instance, so most likely multiple NEM instances will be active at the same time, orchestrated by the UMF coordination. However, the OPEX impact when running multiple NEMs is not simply a summation of the individual results, in the same way that employing a cleaning staff of two does not result in a twice as clean building with a staff of one. This is because 
some of the functionalities and benefits might overlap with each other, and there is a maximum to the total gain that can be achieved. The next section provides a potential solution to NEM aggregation.

TABLE VII. RESULTS OF THE TOY MODEL ANALYSIS FOR PREDICTED OPEX SAVINGS BY SINGLE NEMS

\begin{tabular}{|l|c|c|}
\hline & Min & Max \\
\hline 3: Inter-Cell Interference Coordination (ICC) & $1,3 \%$ & $1,6 \%$ \\
\hline 4: Coverage and Capacity Optimization (CCO) & $3,8 \%$ & $4,6 \%$ \\
\hline 28: Predication-based Load Balancing & $1,2 \%$ & $1,4 \%$ \\
\hline $\begin{array}{l}\text { 32: Self-healing mechanism for Cell Outage } \\
\text { Management (SH) }\end{array}$ & $3,2 \%$ & $3,7 \%$ \\
\hline 34: LTE Load Balancing & $0,8 \%$ & $0,9 \%$ \\
\hline 35: LTE Tilt Optimization & $1,7 \%$ & $2,0 \%$ \\
\hline 46: GP Coverage & $3,5 \%$ & $4,1 \%$ \\
\hline
\end{tabular}

\section{B. NEM Impact Aggregation}

As explained above, since the exact degradation of benefits is not strictly defined, the following NEM order is assumed: NEMs 1 to $n$ are ordered from the largest maximum gain to the smallest maximum gain, and apply for both the best and worse case the following simple formula:

$$
\text { Total OPEX savings }=N E M_{1}+N E M_{2} \times \frac{1}{2}+\cdots+N E M_{n} \times \frac{1}{n}
$$

The ordering is justified by the assumption that an operator will start instantiating the NEMs with the highest expected benefits, and from there one will start looking for incremental improvements. When applying this to the results for this scenario, we have seven NEMs in the following order: (1) NEM 4, (2) NEM 46, (3) NEM 32, (4) NEM 35, (5) NEM 3, (6) NEM 28 and (7) NEM 34. The formula for the best case then looks as follows:

$$
\begin{aligned}
& \text { Best case OPEX savings } \\
& \qquad=4,6 \times \frac{7}{7}+4,1 \times \frac{6}{7}+3,7 \times \frac{5}{7}+2,0 \times \frac{4}{7}+1,6 \times \frac{3}{7}+1,4 \times \frac{2}{7}+0,9 \times \frac{1}{7}
\end{aligned}
$$

whereas the worst case looks as:

$$
\begin{aligned}
& \text { Worst case OPEX savings } \\
& \qquad=3,8 \times \frac{7}{7}+3,5 \times \frac{6}{7}+3,2 \times \frac{5}{7}+1,7 \times \frac{4}{7}+1,3 \times \frac{3}{7}+1,2 \times \frac{2}{7}+0,8 \times \frac{1}{7}
\end{aligned}
$$

resulting in the conclusion that the OPEX savings of this scenario for a typical operator is expected to fall between 11 and 13 per cent.

The development of these ranges is graphically presented in Fig. 2. In this graph, the black lines represent the value calculated by the formulas above. For reference, the grey lines are added, representing the 'simple aggregation' where each additional NEM is considered to have its full autonomous OPEX impact. The solid lines represent the best case, while the dashed lines represent the worst case. Adding additional NEMs will not have a large impact, unless the NEM has a large autonomous gain that will place it at the beginning of the ranking.

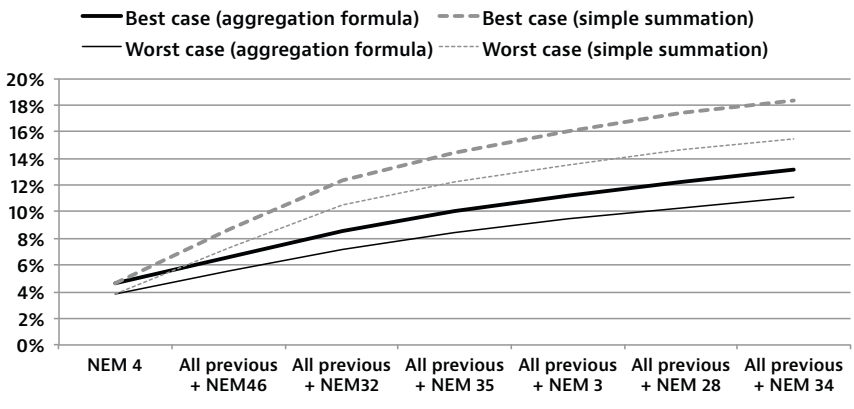

Fig. 2. Progression of the accumulated OPEX impact when more NEMs are added

\section{DISCUSSION AND CONCLUSIONS}

The Toy model, as described in the previous subsections, allowed us to arrive at some quantified results on the OPEX impact of autonomics (UMF and NEMs) for the scenario previously described. However, some positive and negative considerations need to be taken into account regarding the method and its application.

Although a number of market research reports present potential OPEX savings of autonomics, literature does not discuss methods to calculate the OPEX benefits of the implementation of autonomic networking for a typical network operator. This environment is both applied and relying on an unsure future, and is as such more complex than the environments studied by e.g. Verbrugge et al. [10] and Cid et al. [11], which provide static analyses for green-field deployment rather than showing gains. The work proposed in this chapter ventures in new methodological territories for this reason. Our novel method takes into account both expert opinions as well as simulated metrics, and uses established frameworks from literature to make conversion steps.

The model can also yield critical remarks. First of all, our analysis applies to a 'typical' operator, which is a concept that can be discussed. It is unclear whether the typical operator described in the eTOM processes is the same typical operator from Deloitte's OPEX categories. Second, since a typical operator will most likely not exist in the real world, one can expect the framework to be mostly applied by atypical operators, which consequently might alter the experienced OPEX benefits.

Second, one can have considerations regarding some modelling aspects, e.g. the conceptual nature of the requested expert inputs. When experts were asked for mapping tables, it took effort to get them to understand what was actually asked from them - a similar experience as the exercise in which the QFD inputs were gathered. Especially the mapping from metrics to OPEX process flows was considered 'difficult'.

Finally, we can make slight reservations when analysing the output of the model. In Fig. 3, the impacts of the branches $\mathrm{A}$ and $\mathrm{B}$ on the final branch $\mathrm{C}$ are shown. It is striking that for NEM 3 (Inter-Cell Interference Coordination) the expected OPEX gains according to the Toy model turn out negative, which means an expected increase of OPEX. This is due to two significant negative metrics that could not be compensated by two modestly positive metrics. The QFD however does pull 
this NEM to the positive side of the OPEX benefits. One conclusion from this graph is that both lines seem to follow a similar pattern, with an exception for NEM 28, Predictionbased Load Balancing. This indicates that both branches have a shared vision on which NEMs will yield large OPEX gains and which not. The figure also holds another conclusion: with the exception of, again, NEM 28, the impact of branch $\mathrm{A}$ is significantly smaller than that of branch $B$. This can be criticised, since the purpose of the Toy model was to assess the impact of the NEMs and their metrics in another way than the $a$ priori QFD analysis; however, in this exercise, the QFD seems to still have had a large voice in the outcome.

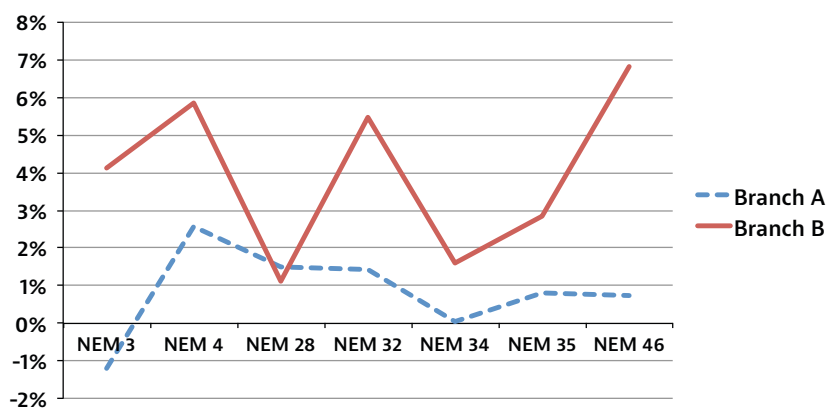

Fig. 3. Impacts of the branches A and B on the outcome of the Toy model

This point is further explored in Fig. 4, which shows the relative impacts between the two branches. Especially NEM 34, LTE Load Balancing, comes out very low on the side of the metric branch. This NEM only provided one metric - one on which it on paper did not even excel greatly. This raises issues with the subjectivity of the NEM metrics and their implementations by the NEM designers. The comparisons between the NEMs performance and the base cases are not always uniform, and moreover, there seems to be an indication that more metrics provided by a NEM designer will favour that particular NEM in terms of expected OPEX benefits.

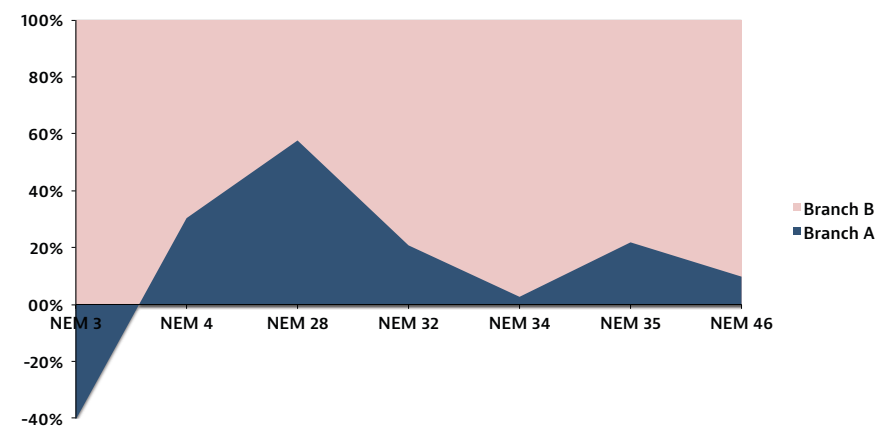

Fig. 4. Relative impacts of branch A and B on the outcome of the Toy model

The Toy model performs an OPEX impact expectation analysis using simulation results as well as expert inputs. Based on the metric results provided by NEM developers, it assesses the impact on eTOM processes. Together with the expert evaluations from a QFD exercise, this allows for an assessment of the impact on the different OPEX categories, and subsequently on the total OPEX for a typical network operator. The results are presented as ranges instead of single figures. In this case, the NEM with the highest results is expected, in isolation and with the UMF active, to deliver between 3,8 and
4,6 per cent OPEX savings typically. In addition, a formula is proposed to assess the total impact of all NEMs of the Scenario together with the UMF. It starts from the most promising NEM, and adds new ones in decreasing order of expected impact. With all NEMs active, the predicted OPEX saving for a typical network operator will fall between 11 and 13 per cent.

\section{ACKNOWLEDGMENT}

The research leading to these results has been performed within the UniverSelf project (www.univerself-project.eu) and received funding from the European Community's Seventh Framework Programme (FP7/2007-2013) under grant agreement $n^{\circ} 257513$.

\section{REFERENCES}

[1] FP7/ICT project UniverSelf, (ICT-2009-257513), Sep. 2010- Aug. 2013, Website: http://www.univerself-project.eu, Dec. 2012.

[2] S. Spek, V. Gonçalves, S. Delaere, "Business Impact Assessment for Autonomic Network Management: Identifying Business Impact Indicators From Use Case Requirements," in Sixth International Conference on Mobile Ubiquitous Computing, Systems, Services and Technologies (UBICOMM), 2012.

[3] UniverSelf use case 4, SON and SON collaboration according to operator policies - case study part 1", available at http://www.univerself-project.eu/white-papers.

[4] R. Combes, Z. Altman and E. Altman, "Self-Organizing Relays: Dimensioning, Self-Optimization and Learning", IEEE Transactions on Network Management, TNSM, vol. 9, pp. 487-500, Dec. 2012.

[5] G. Athanasiou, K. Tsagkaris, D. Karvounas, P. Vlacheas, P. Demestichas, "Multi-Objective Traffic Engineering for Future Networks," Communications Letters, IEEE, vol.16, no.1, pp.101-103, January 2012.

[6] R. Combes, Z. Altman and E. Altman, "Coordination of autonomic functionalities in communications networks," WiOpt 2013, Tsukuba Science City, Japan.

[7] TM Forum, Business Process Framework (eTOM), GB921 Addendum E: Application Note: End-to-End Business Flows, Release 9.1, TM Forum Approved Version 9.4, April, 2011.

[8] L. K. Chan and M. L. Wu, "Quality function deployment: A literature review," European Journal of Operational Research, vol. 143, no. 3, pp. 463-497, 2002.

[9] B. Fuentez (ed.), "UniverSelf Deliverable 4.15 - Synthetic analysis of deployment results and impacts", public project deliverable, November 2013. http://www.univerself-project.eu/technical-reports

[10] S. Verbrugge, S. Pasqualini, F.-J. Westphal, M. Jäger, A. Iselt, A. Kirstädter, R. Chahine, D. Colle, M. Pickavet, and P. Demeester, "Modeling operational expenditures for telecom operators," in 9th Conference on Optical Network Design \& Modelling (ONDM 2005), Milan, Italy, 2005.

[11] C. Cid, M. Ruiz, L. Velasco, and G. Junyent, "Costs and Revenues Models for Optical Networks Architectures Comparison."

[12] "Motorola LTE Self Organizing Networks. Motorola's revolutionairy SON solution for LTE OPEX reduction," Motorola, 2009.

[13] J. Buvat and S. Basu, "Quest for Margins: Operational Cost Strategies for Mobile Operators in Europe," Capgemini, 42, 2009.

[14] J. Harno, "Techno-economic analysis of beyond 3G mobile technology alternatives," info, vol. 11, no. 3, pp. 45-63, 2009.

[15] J. Bernard, T. Broschuk, P. Doane, M. Jadoul, and M. Nespatti, "Optimization. Secrets to network success: small changes deliver big results," Alcatel-Lucent, 2010.

[16] H. Schwarz and M. Schmitz, "Green Gold. How energy savings are given due consideration in outsourcing agreements," DMR, Magazine for Management and Technology, vol. 4, 2011.

[17] M. Locker, L. Glover, and E. Heisler, "The seven cost management principles for wireless carriers: When your average cost reduction program just won't do," Deloitte, 2011. 